MS7-P4 Crystal structure of the soluble domain of RC1339/APRc from Rickettsia conorii, a retropepsin-like aspartic protease

\author{
Alla Gustchina ${ }^{1}, \mathrm{Mi} \mathrm{Li}^{1,2}$, Rui $\mathrm{Cruz}^{3,4}$, Marisa Simões ${ }^{3,4}$, Pedro \\ Curto $^{3,5}$, Juan Martinez ${ }^{5}$, Carlos Faro ${ }^{3,4}$, Isaura Simões ${ }^{3,4}$, \\ Alexander Wlodawer ${ }^{1}$
}

1. Macromolecular Crystallography Laboratory, National Cancer Institute, Frederick, MD, USA

2. Basic Science Program, Leidos Biomedical Research, Frederick National Laboratory for Cancer Research, Frederick, MD, USA

3. Center for Neuroscience and Cell Biology, University of Coimbra, Coimbra, Portugal

4. Biocant, Biotechnology Innovation Center, Cantanhede, Portugal 5. Vector-Borne Diseases Laboratories, School of Veterinary Medicine, Louisiana State University, Baton Rouge, LA, USA

email: gustchia@mail.nih.gov

The common secondary structure template among domains/monomers of pepsin-like aspartic proteases and retropepsins supports the view that these proteases are evolutionarily related, and that pepsins may have arisen by gene duplication and fusion of an ancestral form of retropepsins. The nature of this primordial single-lobed aspartic protease has been the matter of debate over the years, mostly due to the lack of compelling evidence for the presence of these enzymes in prokaryotes. However, this argument has been first challenged with finding pepsin homologs in a restricted number of bacteria and the observation that at least one of these genes encodes an active enzyme. More recently, we reported the identification of a gene coding for a membrane-embedded, single-lobed aspartic protease, highly conserved in the genomes of 55 species of Rickettsia. Using Rickettsia conorii gene homolog rc1339, we provided evidence that the encoded product (APRc), indeed shares several enzymatic properties with viral retropepsins (Cruz, et al., PLoS Pathog., 10, e1004324, 2014)

These resemblance of enzymatic features suggested that APRc might indeed represent a more primordial form of retropepsins. In this work, crystal structures of two constructs of the soluble domain of RC1339/APRc from Rickettsia conorii have been determined in three different crystal forms. The results clearly show that the fold of APRc monomer is closely related to the fold of retropepsins, but the quaternary structure of the dimer differed from the canonical retropepsins. The observed dimer is most likely an artefact of expression and/or crystallization since it cannot support the previously reported enzymatic activity of APRc.

Overall, our results support the concepts that APRc may indeed represent a putative common ancestor of monomeric and dimeric aspartic proteases, as well as possible existence of a different evolutionary pathway for these enzymes.

\section{MS7-P5 Purification of Z.mobilis \\ levansucrase for structural studies}

Burcu Kaplan Türköz ${ }^{1}$, Dicle Dilara Akpınar ${ }^{1}$, Filiz Döner ${ }^{1}$

1. Ege University, Faculty of Engineering, Department of Food Engineering, Bornova, İzmir, Turkey

email: bkaplan@sabanciuniv.edu

Levansucrases (Lsc) are enzymes, which have both sucrose hydrolysis and transfructosylation activity. Several microorganisms produce the enzyme, which have different product specificities. Among those identified from Gram-negative bacteria preferably produce short chain fructans; fructooligosaccharides (FOS). Gram-positive Lsc, on the other hand, are shown to produce mainly levan, long fructan polymers (1). Levan is a valuable polysaccharide used in food, biomedical and chemical industries (2). FOS are used as low calorie sweeteners and were shown to be effective prebiotics (3). Lsc produced by the gram-negative Zymomonas mobilis was shown to produce both FOS and levan depending on the reaction conditions such as substrate concentation and temperature (4). In order to understand the molecular details of this dual product formation ability, we are interested in solving the atomic structure of the native enzyme. To achieve this goal, we first optimized Z.mobilis growth conditions to maximize the production of the extracellular Lsc (5). In this study, the extracellular enzyme will be purified and crystallized for structural studies. Lsc was concentrated from the high volume cell free supernatant by salting out and active enzyme was obtained. This crude enzyme will be further purified by ion exchange and size exclusion chromatography. The crystallization of purified Lsc will be investigated using crystallization screens and in the presence of different substrate concentrations. Obtaning the crystal structure of this unique enzyme will allow us to compare its structural differences with only levan or only FOS producing levansucrases. The results might help understand the details of the enzyme's ability to produce both fructans and give valuable information for targeted enzyme design to increase the production of the desired polymer; levan or FOS.

Acknowledgements: This work is supported by TÜBİTAK-TOVAG (grant number:214O174).

References: 1. Wuerges, J., et al. 2015, Journal of Structural Biology, 191(3), 290-298. 2. Srikanth, R., 2015, Carbohydrate Polymers, 120, 102-14. 3. Sangeetha, P. T., 2005, Trends in Food Science \& Technology, 16(10), 442-457. 4. Santos-Moriano, P.,2015, Journal of Molecular Catalysis B: Enzymatic, 119, 18-25. 5. Erdal,Ö., 2016, M.Sc. Thesis, Ege University (TR)

Keywords: Levansucrase, Levan, Fructooligosaccharides, Crystallization 